\title{
Some Properties on Degrees of Dominating Sets for Circular-Arc Graph
}

\author{
Dr. A. Sudhakaraiah*, T. Visalakshi ${ }^{1}$ ，Dr. T. Venkateswarlu ${ }^{2}$, Dr. K. Narayana ${ }^{3}$ \\ *Associate professor, Department of Mathematics, S.V.University, Tirupati, A.P., India. \\ 1Research Scholar, Department of Mathematics, S.V.University, Tirupati, A.P., India. \\ 2 Lecturer, Department of Mathematics, V.R.College, Nellore, A.P., India. \\ 3 Assistant professor, Department of Mathematics, Geethanjali Institute of Science and Technology, Nellore, India.
}

\begin{abstract}
Domination in graphs has applications to several fields. A similar problem occurs when the maximum distance to a facility is fixed and one attempts to minimize the no of facilities necessary so that everyone is serviced. The prominence of Circular-arc graphs in the current times is evident from the enhanced research focus and attempts for extensive exploration during the recent years. In this paper, we derived the some properties on sum of degrees of elements in a minimum dominating set through the quotient of divisor 3 , domination number and the number of vertices of the circular-arc graphs corresponding to a circular-arc family in a particular case of each arc intersects the next arc only by using Euclidean division lemma.
\end{abstract}

Keywords: Circular-arc family, circular-arc graph, minimum dominating set, domination number, Euclidean division lemma.

\section{INTRODUCTION}

Graph theory is a fast developing area in mathematics with a wide range of applications including computer science. Diverse domains such as linguistics, physical sciences, social sciences, communication engineering and several others make use of the Graph Theory. Further, it plays a prominent role specifically in computer applications pertaining to switching theory, artificial intelligence, logic design, operating systems, formal languages, compiler writing, computer graphics and organization and retrieval of information. Today, graph theory has developed into a specific discipline on its own. The emergence and development of computers and their applications have immensely contributed to the enhanced applications of graph theory. Several branches in mathematics commence with sets and the relations, with an exception of graph theory.

\section{PRELIMINARIES}

All graphs considered in this paper are finite, undirected, with no loops or multiple edges. A graph is said to be a simple graph if it has no loops and no parallel edges. If a vertex $\mathrm{v} \in \mathrm{V}$ in a graph $\mathrm{G}$ is one of the extreme vertices of any edge $\mathrm{e} \in \mathrm{E}$ then the edge ' $\mathrm{e}$ ' and the vertex ' $\mathrm{v}$ ' are said be incident with each other. The number of edges incident with a vertex $\mathrm{V}$ is called degree of $\mathrm{V}$ and is denoted by $\operatorname{deg}(\mathrm{v})$. If $\operatorname{deg}(\mathrm{v})=1$ then the vertex $\mathrm{v}$ is said to be a pendent vertex. If $\operatorname{deg}(\mathrm{v})=0$ then the vertex $v$ is said to be an isolated vertex. Let $G$ be a graph, a set $\mathrm{D} \subseteq \mathrm{V}$ is a dominating set [1] of $\mathrm{G}$ if every vertex in $\mathrm{V} \backslash \mathrm{D}$ is adjacent to some vertex in $\mathrm{D}$. The domination number[1][2] $\gamma(\mathrm{G})$ of $\mathrm{G}$ is the minimum cardinality of a dominating set.

\section{PRE-REQUISITE RESULTS}

\subsection{RESULT}

Let $\quad \mathrm{A}=\left\{\mathrm{A}_{1}, \mathrm{~A}_{2}, \mathrm{~A}_{3}, \ldots \ldots \ldots . . . \mathrm{A}_{v}\right\}, \forall \mathrm{v} \geq 3 \quad$ be any finite circular-arc family such that every arc intersects the next arc only. Let $\mathrm{G}$ be a circular-arc graph corresponding to a circular-arc family A. Suppose $v=3 a+b$, where $\mathrm{b}=0,1,2$ and $\mathrm{a}$ is any positive integer. If $\mathrm{b}=0$ then the minimum dominating set is either $\mathrm{D}=\left\{\mathrm{v}_{3 \mathrm{i}-2} / \mathrm{i} \in \mathrm{N}, 1 \leq \mathrm{i} \leq \mathrm{a}\right\}$ or $\mathrm{D}=\left\{\mathrm{v}_{3 \mathrm{i}-1} / \mathrm{i} \in \mathrm{N}, 1 \leq \mathrm{i} \leq \mathrm{a}\right\}$ or $\mathrm{D}=\left\{\mathrm{v}_{3 \mathrm{i}} / \mathrm{i} \in \mathrm{N}, 1 \leq \mathrm{i} \leq \mathrm{a}\right\}$ and the domination number $\gamma=\mathrm{a}$.

\subsection{RESULT}

If $\mathrm{G}$ be a circular-arc graph to a circular-arc family $\mathrm{A}=\left\{\mathrm{A}_{1}, \mathrm{~A}_{2}, \mathrm{~A}_{3}, \ldots \ldots \ldots . . . \mathrm{A}_{\mathrm{v}}\right\}, \forall \mathrm{v} \geq 3 \quad$ in which every arc intersects the next arc only. Suppose $v=3 a+b$, where $b=0,1,2$ and $a$ is any positive integer. If $b=1$ then the minimum dominating set is $\mathrm{D}=\left\{\mathrm{v}_{3 \mathrm{i}-1}, \mathrm{v}_{\mathrm{v}} / \mathrm{i} \in \mathrm{N}, 1 \leq \mathrm{i} \leq \mathrm{a}\right\}$ and the domination number $\gamma=\mathrm{a}+1$.

\subsection{RESULT}

Let $\mathrm{A}=\left\{\mathrm{A}_{\mathrm{i}} / \mathrm{i} \in \mathrm{N}, 1 \leq \mathrm{i} \leq \mathrm{v}\right\}, \forall \mathrm{v} \geq 3$ be any finite circulararc family in which every arc intersects the next arc only and $\mathrm{G}$ be a circular-arc graph corresponding to a circular-arc family A. Suppose $v=3 a+b$ where $b=0,1,2$ and $a$ is any 
positive integer. If $b=2$ then the minimum dominating set is either

$$
\mathrm{D}=\left\{\mathrm{v}_{3 \mathrm{i}-1}, \mathrm{v}_{\mathrm{v}-1} / \mathrm{i} \in \mathrm{N}, 1 \leq \mathrm{i} \leq \mathrm{a}\right\}
$$
$\mathrm{D}=\left\{\mathrm{v}_{3 \mathrm{i}-1}, \mathrm{v}_{\mathrm{v}} / \mathrm{i} \in \mathrm{N}, 1 \leq \mathrm{i} \leq \mathrm{a}\right\}$ and the domination number $\gamma=\mathrm{a}+1$.

\subsection{RESULT}

Let $\mathrm{A}=\left\{\mathrm{A}_{1}, \mathrm{~A}_{2}, \mathrm{~A}_{3}, \ldots \ldots \ldots, \mathrm{A}_{\mathrm{v}}\right\}, \forall v \geq 3$ be any finite circular-arc family such that every arc intersects the next arc only and let $\mathrm{G}$ be a circular-arc graph corresponding to a circular-arc family $\mathrm{A}$ if there is one-to-one correspondence between the vertex set $\mathrm{V}$ and circular-arc family $\mathrm{A}$ where $\mathrm{V}=\mathrm{v}_{1}, \mathrm{v}_{2}, \mathrm{v}_{3}, \ldots \ldots \ldots, \mathrm{v}_{v}$ then $\operatorname{deg}\left(\mathrm{v}_{1}\right)=\operatorname{deg}\left(\mathrm{v}_{2}\right)==$

$=\operatorname{deg}\left(v_{n}\right)=2$.

\section{MAIN THEOREMS AND PRACTICAL PROBLEMS}

\subsection{THEOREM}

Let $\mathrm{A}=\left\{\mathrm{A}_{1}, \mathrm{~A}_{2}, \mathrm{~A}_{3}, \mathrm{~A}_{4}, \ldots \ldots \ldots, \mathrm{A}_{v}\right\}, \forall v \geq 3$ be any finite circular-arc family such that every arc $A_{i}$ intersects the next arc only and let $\mathrm{G}$ be a circular-arc graph corresponding to a circular-arc family A. Suppose $v=3 a+b$, where $b=0,1,2$ and $a$ is any positive integer. If $b=0$ then three cases will arise.

Case (i): The minimum dominating set $\mathrm{D}=\left\{\mathrm{v}_{3 \mathrm{i}-2} / \mathrm{i} \in \mathrm{N}, 1 \leq \mathrm{i} \leq \mathrm{a}\right\}$ and $\gamma=\mathrm{a}$ and then the following deductions are true (i) $\sum_{\mathrm{v} \in \mathrm{D}} \operatorname{deg}(\mathrm{v})=2 \mathrm{a}(\mathrm{ii}) \sum_{\mathrm{v} \in \mathrm{D}} \operatorname{deg}(\mathrm{v})=2 \gamma$

(iii) $\sum_{\mathrm{v} \in \mathrm{D}} \operatorname{deg}(\mathrm{v})=\frac{2 \mathrm{v}}{3}$

Case (ii): The minimum dominating set $\mathrm{D}=\left\{\mathrm{v}_{3 \mathrm{i}-1} / \mathrm{i} \in \mathrm{N}, 1 \leq \mathrm{i} \leq \mathrm{a}\right\}$ and $\gamma=\mathrm{a}$ and then the following deductions are true $($ i $) \sum_{v \in D} \operatorname{deg}(v)=2 a($ ii $) \sum_{v \in D} \operatorname{deg}(v)=2 \gamma$

(iii) $\sum_{\mathrm{v} \in \mathrm{D}} \operatorname{deg}(\mathrm{v})=\frac{2 \mathrm{v}}{3}$

Case (iii): The minimum dominating set $\mathrm{D}=\left\{\mathrm{v}_{3 \mathrm{i}} / \mathrm{i} \in \mathrm{N}, 1 \leq \mathrm{i} \leq \mathrm{a}\right\}$ and $\gamma=\mathrm{a}$ and then the following deductions are true $(\mathrm{i}) \sum_{\mathrm{v} \in \mathrm{D}} \operatorname{deg}(\mathrm{v})=2 \mathrm{a}\left(\right.$ ii) $\sum_{\mathrm{v} \in \mathrm{D}} \operatorname{deg}(\mathrm{v})=2 \gamma$ (iii) $\sum_{\mathrm{v} \in \mathrm{D}} \operatorname{deg}(\mathrm{v})=\frac{2 \mathrm{v}}{3}$

\section{PROOF:}

Let $\mathrm{A}=\left\{\mathrm{A}_{1}, \mathrm{~A}_{2}, \mathrm{~A}_{3}, \mathrm{~A}_{4}, \ldots \ldots \ldots, \mathrm{A}_{\mathrm{v}}\right\}, \forall v \geq 3$ be any finite circular-arc family such that every arc $A_{i}$ intersects the next arc only and let $\mathrm{G}$ be a circular-arc graph corresponding to a circular-arc family A if there is one-to-one correspondence between the vertex set $\mathrm{V}$ and circular-arc family $\mathrm{A}$, where $\mathrm{V}=\mathrm{v}_{1}, \mathrm{v}_{2}, \mathrm{v}_{3}, \ldots \ldots ., \mathrm{v}_{v}$

Suppose $v=3 a+b$, where $b=0,1,2$ by Euclidean division lemma,

If $b=0$, then $v=3 a+0 \Rightarrow v=3 a \Rightarrow a=\frac{v}{3}$

Then three cases will arise

Case(i): we have $\mathrm{D}=\left\{\mathrm{v}_{3 \mathrm{i}-2} / \mathrm{i} \in \mathrm{N}, 1 \leq \mathrm{i} \leq \mathrm{a}\right\}$ and the domination number $\gamma=\mathrm{a}$

$$
\begin{aligned}
& \text { i.e., } D=v_{1}, v_{4}, v_{7}, \ldots \ldots \ldots . ., v_{3 a-2} \\
& \text { i.e., } \mathrm{D}=\mathrm{v}_{1}, \mathrm{v}_{4}, \mathrm{v}_{7}, \ldots \ldots \ldots \ldots, \mathrm{v}_{\mathrm{v}-2} \\
& \text { (i) } \sum_{\mathrm{v} \in \mathrm{D}} \operatorname{deg}(\mathrm{v})=\operatorname{deg}\left(\mathrm{v}_{1}\right)+\operatorname{deg}\left(\mathrm{v}_{4}\right)+\operatorname{deg}\left(\mathrm{v}_{7}\right)+\cdots+\operatorname{deg}\left(\mathrm{v}_{v-2}\right) \\
& =2+2+2+\cdots \cdots \cdots+2 \\
& =2 \mathrm{a}
\end{aligned}
$$$$
\therefore \sum_{\mathrm{v} \in \mathrm{D}} \mathrm{deg}(\mathrm{v})=2 \mathrm{a}
$$

(ii) $\sum_{\mathrm{v} \in \mathrm{D}} \operatorname{deg}(\mathrm{v})=2 \mathrm{a}=2 \gamma \quad(\because \gamma=\mathrm{a})$

(iii) $\sum_{\mathrm{v} \in \mathrm{D}} \operatorname{deg}(\mathrm{v})=2 \mathrm{a}=2\left(\frac{v}{3}\right)=\frac{2 v}{3} \quad\left(\because \mathrm{a}=\frac{v}{3}\right)$

Case(ii): we have $\mathrm{D}=\left\{\mathrm{v}_{3 \mathrm{i}-1} / \mathrm{i} \in \mathrm{N}, 1 \leq \mathrm{i} \leq \mathrm{a}\right\}, \gamma=\mathrm{a}$

$$
\begin{aligned}
& \text { i.e., } D=v_{2}, v_{5}, v_{8}, \ldots \ldots \ldots \ldots, v_{3 a-1} \\
& \text { i.e., } \mathrm{D}=\mathrm{v}_{2}, \mathrm{v}_{5}, \mathrm{v}_{8}, \ldots \ldots \ldots \ldots, \mathrm{v}_{\mathrm{v}-1} \\
& \text { (i) } \sum_{\mathrm{v} \in \mathrm{D}} \operatorname{deg}(\mathrm{v})=\operatorname{deg}\left(\mathrm{v}_{2}\right)+\operatorname{deg}\left(\mathrm{v}_{5}\right)+\cdots \cdots+\operatorname{deg}\left(\mathrm{v}_{\mathrm{v}-1}\right) \\
& =2+2+2+\cdots \cdots \cdots+2 \\
& =2(1+1+1+\cdots \cdots+\cdots+1) \\
& =2 \mathrm{a} \\
& \therefore \sum_{\mathrm{v} \in \mathrm{D}} \operatorname{deg}(\mathrm{v})=2 \mathrm{a} \\
& \text { (ii) } \sum_{\mathrm{v} \in \mathrm{D}} \operatorname{deg}(\mathrm{v})=2 \mathrm{a}=2 \gamma \quad(\because \mathrm{a}=\gamma) \\
& \text { (iii) } \sum_{\mathrm{v} \in \mathrm{D}} \operatorname{deg}(\mathrm{v})=2 \mathrm{a}=2\left(\frac{v}{3}\right)=\frac{2 v}{3}\left(\because \mathrm{a}=\frac{v}{3}\right)
\end{aligned}
$$

Case(iii): we have $\mathrm{D}=\left\{\mathrm{v}_{3 \mathrm{i}} / \mathrm{i} \in \mathrm{N}, 1 \leq \mathrm{i} \leq \mathrm{a}\right\}, \gamma=\mathrm{a}$

$$
\begin{aligned}
& \text { i.e., } D=v_{3}, v_{6}, v_{9}, \ldots \ldots \ldots . ., v_{3 a} \\
& \text { i.e., } D=v_{3}, v_{6}, v_{9}, \ldots \ldots \ldots . . ., v_{v}
\end{aligned}
$$


(i) $\sum_{\mathrm{v} \in \mathrm{D}} \operatorname{deg}(\mathrm{v})=\operatorname{deg}\left(\mathrm{v}_{3}\right)+\operatorname{deg}\left(\mathrm{v}_{6}\right)+\cdots \cdots \cdots+\operatorname{deg}\left(\mathrm{v}_{v}\right)$

$$
\begin{aligned}
& =2+2+2+\cdots \cdots \cdots+2 \\
& =2(1+1+1+\cdots \cdots \cdots+1) \\
& =2 \mathrm{a}
\end{aligned}
$$

$\therefore \sum_{\mathrm{v} \in \mathrm{D}} \operatorname{deg}(\mathrm{v})=2 \mathrm{a}\left(\right.$ ii) $\sum_{\mathrm{v} \in \mathrm{D}} \operatorname{deg}(\mathrm{v})=2 \mathrm{a}=2 \gamma \quad(\because \mathrm{a}=\gamma)$

(iii) $\sum_{\mathrm{v} \in \mathrm{D}} \operatorname{deg}(\mathrm{v})=2 \mathrm{a}=2\left(\frac{v}{3}\right)=\frac{2 v}{3} \quad\left(\because \mathrm{a}=\frac{v}{3}\right)$

Hence theorem is hold.

\section{PRACTICAL PROBLEM:}

Let $A=\left\{A_{1}, A_{2}, A_{3}, A_{4}, A_{5}, A_{6}, A_{7}, A_{8}, A_{9}\right\} \quad$ be any circular-arc family and let $G$ be a circular-arc graph corresponding to a circular-arc family $\mathrm{A}$ is as follows

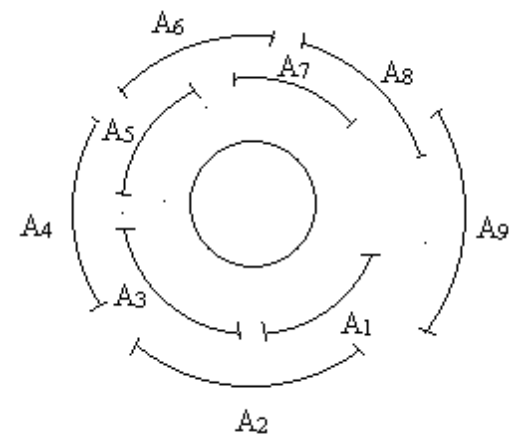

Fig. 3.1 Circular-arc family

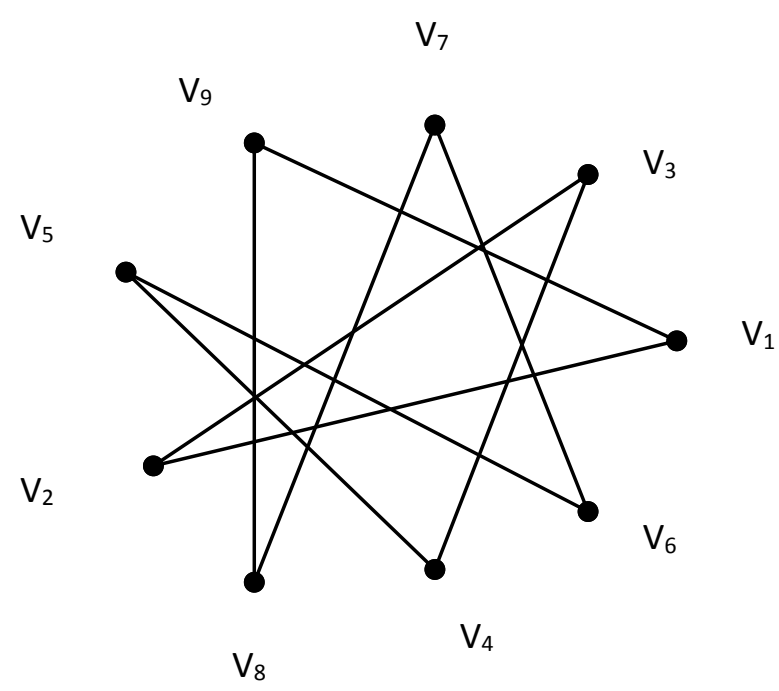

Fig.3.2 Circular-arc graph
Given circular-arc family A, there are nine arcs in which every arc intersects the next arc only.

then $v=9=3 \times 3+0$

This is of the form $v=3 a+b$, here $a=3$ and $b=0$.

If $b=0$ then three cases will arise

Case(i): The minimum dominating set

$\mathrm{D}=\left\{\mathrm{v}_{3 \mathrm{i}-2} / \mathrm{i} \in \mathrm{N}, 1 \leq \mathrm{i} \leq \mathrm{a}\right\}$

$\mathrm{D}=\left\{\mathrm{v}_{3 \mathrm{i}-2} / \mathrm{i} \in \mathrm{N}, 1 \leq \mathrm{i} \leq 3\right\}$

$\mathrm{D}=\left\{\mathrm{v}_{1}, \mathrm{v}_{4}, \mathrm{v}_{7}\right\}$ and $\gamma=3$.

Now $\sum_{\mathrm{v} \in \mathrm{D}} \operatorname{deg}(\mathrm{v})=\operatorname{deg}\left(\mathrm{v}_{1}\right)+\operatorname{deg}\left(\mathrm{v}_{4}\right)+\operatorname{deg}\left(\mathrm{v}_{7}\right)$ $=2+2+2$

$$
\therefore \sum_{\mathrm{v} \in \mathrm{D}} \operatorname{deg}(\mathrm{v})=6
$$

and (i) $\sum_{\mathrm{v} \in \mathrm{D}} \operatorname{deg}(\mathrm{v})=2 \mathrm{a}=2 \times 3=6 \quad(\because \mathrm{a}=3)$

(ii) $\sum_{\mathrm{v} \in \mathrm{D}} \operatorname{deg}(\mathrm{v})=2 \gamma=2 \times 3=6 \quad(\because \gamma=3)$

(iii) $\sum_{\mathrm{v} \in \mathrm{D}} \operatorname{deg}(\mathrm{v})=\frac{2 \mathrm{v}}{3}=\frac{2 \times 9}{3}=\frac{18}{3}=6 \quad(\because v=9)$

Case (ii): The minimum dominating set is

$$
\begin{aligned}
& \mathrm{D}=\left\{\mathrm{v}_{3 \mathrm{i}-1} / \mathrm{i} \in \mathrm{N}, 1 \leq \mathrm{i} \leq \mathrm{a}\right\} \\
& \mathrm{D}=\left\{\mathrm{v}_{3 \mathrm{i}-1} / \mathrm{i} \in \mathrm{N}, 1 \leq \mathrm{i} \leq 3\right\} \\
& \mathrm{D}=\left\{\mathrm{v}_{2}, \mathrm{v}_{5}, \mathrm{v}_{8}\right\} \text { and } \gamma=3 .
\end{aligned}
$$

Now $\sum_{\mathrm{v} \in \mathrm{D}} \operatorname{deg}(\mathrm{v})=\operatorname{deg}\left(\mathrm{v}_{2}\right)+\operatorname{deg}\left(\mathrm{v}_{5}\right)+\operatorname{deg}\left(\mathrm{v}_{8}\right)$

$$
=2+2+2
$$

$\therefore \sum_{\mathrm{v} \in \mathrm{D}} \operatorname{deg}(\mathrm{v})=6$

In this case also we have observed the following

(i) $\sum_{\mathrm{v} \in \mathrm{D}} \operatorname{deg}(\mathrm{v})=2 \mathrm{a}($ ii) $) \sum_{\mathrm{v} \in \mathrm{D}} \operatorname{deg}(\mathrm{v})=2 \gamma($ iii $) \sum_{\mathrm{v} \in \mathrm{D}} \operatorname{deg}(\mathrm{v})=\frac{2 \mathrm{v}}{3}$.

Case (iii): The minimum dominating set is

$$
\begin{aligned}
& \mathrm{D}=\left\{\mathrm{v}_{3 \mathrm{i}} / \mathrm{i} \in \mathrm{N}, 1 \leq \mathrm{i} \leq \mathrm{a}\right\} \\
& \mathrm{D}=\left\{\mathrm{v}_{3 \mathrm{i}} / \mathrm{i} \in \mathrm{N}, 1 \leq \mathrm{i} \leq 3\right\} \\
& \mathrm{D}=\left\{\mathrm{v}_{3}, \mathrm{v}_{6}, \mathrm{v}_{9}\right\} \text { and } \gamma=3 . \\
& \text { Now } \sum_{\mathrm{v} \in \mathrm{D}} \operatorname{deg}(\mathrm{v})=\operatorname{deg}\left(\mathrm{v}_{3}\right)+\operatorname{deg}\left(\mathrm{v}_{6}\right)+\operatorname{deg}\left(\mathrm{v}_{9}\right) \\
& \therefore \sum_{\mathrm{v} \in \mathrm{D}} \operatorname{deg}(\mathrm{v})=6
\end{aligned}
$$


In this case also we have observed the following

(i) $\sum_{\mathrm{v} \in \mathrm{D}} \operatorname{deg}(\mathrm{v})=2 \mathrm{a}\left(\right.$ ii) $\sum_{\mathrm{v} \in \mathrm{D}} \operatorname{deg}(\mathrm{v})=2 \gamma($ iii $) \sum_{\mathrm{v} \in \mathrm{D}} \operatorname{deg}(\mathrm{v})=\frac{2 \mathrm{v}}{3}$.

Thus, the theorem is verified.

\subsection{THEOREM}

If $G$ be a circular-arc graph to a circular-arc family $\mathrm{A}=\left\{\mathrm{A}_{1}, \mathrm{~A}_{2}, \mathrm{~A}_{3}, \cdots \ldots \ldots . . . \mathrm{A}_{v}\right\}, \forall \mathrm{v} \geq 3 \quad$ in which every arc intersects the next arc only. Suppose $v=3 a+b$, where $b=0,1,2$ and $a$ is any positive integer. If $b=1$ then the minimum dominating set is $\mathrm{D}=\left\{\mathrm{v}_{3 \mathrm{i}-1}, \mathrm{v}_{\mathrm{v}} / \mathrm{i} \in \mathrm{N}, 1 \leq \mathrm{i} \leq \mathrm{a}\right\}$ and the domination number $\gamma=\mathrm{a}+1$ and then the following deductions are true
(i) $\sum_{v \in D} \operatorname{deg}(v)=2 a+2$
(ii) $\sum_{\mathrm{v} \in \mathrm{D}} \operatorname{deg}(\mathrm{v})=2 \gamma$

(iii) $\sum_{v \in D} \operatorname{deg}(v)=\frac{2 v+4}{3}$.

\section{PROOF:}

Let $\mathrm{A}=\left\{\mathrm{A}_{1}, \mathrm{~A}_{2}, \mathrm{~A}_{3}, \mathrm{~A}_{4}, \ldots \ldots \ldots, \mathrm{A}_{\mathrm{v}}\right\}, \forall v \geq 3$ be any finite circular-arc family such that every arc $A_{i}$ intersects the next arc only and let $\mathrm{G}$ be a circular-arc graph corresponding to a circular-arc family $\mathrm{A}$ if there is one-to-one correspondence between the vertex set $\mathrm{V}$ and circular-arc family $\mathrm{A}$, where $\mathrm{V}=\mathrm{v}_{1}, \mathrm{v}_{2}, \mathrm{v}_{3}, \ldots \ldots ., \mathrm{v}_{v}$

Suppose $v=3 a+b$, where $b=0,1,2$ by Euclidean division lemma

If $b=1$, then $v=3 a+1 \Rightarrow v-1=3 a \Rightarrow a=\frac{v-1}{3}$

Then we have $\mathrm{D}=\left\{\mathrm{v}_{3 \mathrm{i}-1}, \mathrm{v}_{\mathrm{v}} / \mathrm{i} \in \mathrm{N}, 1 \leq \mathrm{i} \leq \mathrm{a}\right\}$

and $\gamma=\mathrm{a}+1$

i.e., $\mathrm{D}=\mathrm{v}_{2}, \mathrm{v}_{5}, \mathrm{v}_{8}, \ldots \ldots \ldots \ldots, \mathrm{v}_{3 \mathrm{a}-1}, \mathrm{v}_{v}$

i.e., $\mathrm{D}=\mathrm{v}_{2}, \mathrm{v}_{5}, \mathrm{v}_{8}, \ldots \ldots \ldots . ., \mathrm{v}_{v-2}, \mathrm{v}_{v}$

(i) $\sum_{\mathrm{v} \in \mathrm{D}} \operatorname{deg}(\mathrm{v})=\operatorname{deg}\left(\mathrm{v}_{2}\right)+\operatorname{deg}\left(\mathrm{v}_{5}\right)+\cdots \cdots+\operatorname{deg}\left(\mathrm{v}_{\mathrm{v}}\right)$

$$
\begin{aligned}
& =2+2+2+\cdots \cdots \cdots+2+2 \\
& =2(1+1+1+\cdots \cdots \cdot \cdots+1) \\
& =2(a+1) \\
& =2 a+2
\end{aligned}
$$

$\therefore \sum_{\mathrm{v} \in \mathrm{D}} \mathrm{deg}(\mathrm{v})=2 \mathrm{a}+2$ (ii) $\sum_{\mathrm{v} \in \mathrm{D}} \operatorname{deg}(\mathrm{v})=2 \mathrm{a}+2$

$$
\begin{aligned}
& =2(\gamma-1)+2 \quad(\because \gamma=\mathrm{a}+1) \\
& =2 \gamma-2+2 \\
& =2 \gamma \\
\therefore \sum_{\mathrm{v} \in \mathrm{D}} \operatorname{deg}(\mathrm{v}) & =2 \gamma \\
(\text { iii) }) \sum_{\mathrm{v} \in \mathrm{D}} \operatorname{deg}(\mathrm{v}) & =2 \mathrm{a}+2 \\
& =2\left(\frac{v-1}{3}\right)+2 \quad\left(\because \mathrm{a}=\frac{v-1}{3}\right) \\
& =\frac{2 v-2}{3}+2 \\
& =\frac{2 v-2+6}{3} \\
& =\frac{2 v+4}{3} \\
\therefore \sum_{\mathrm{v} \in \mathrm{D}} \operatorname{deg}(\mathrm{v}) & =\frac{2 v+4}{3}
\end{aligned}
$$

\section{PRACTICAL PROBLEM}

Let $\mathrm{A}=\left\{\mathrm{A}_{1}, \mathrm{~A}_{2}, \mathrm{~A}_{3}, \mathrm{~A}_{4}, \mathrm{~A}_{5}, \mathrm{~A}_{6}, \mathrm{~A}_{7}, \mathrm{~A}_{8}, \mathrm{~A}_{9}, \mathrm{~A}_{10}\right\}$ be any circular-arc family and let $G$ be a circular-arc graph corresponding to a circular-arc family $\mathrm{A}$ is as follow

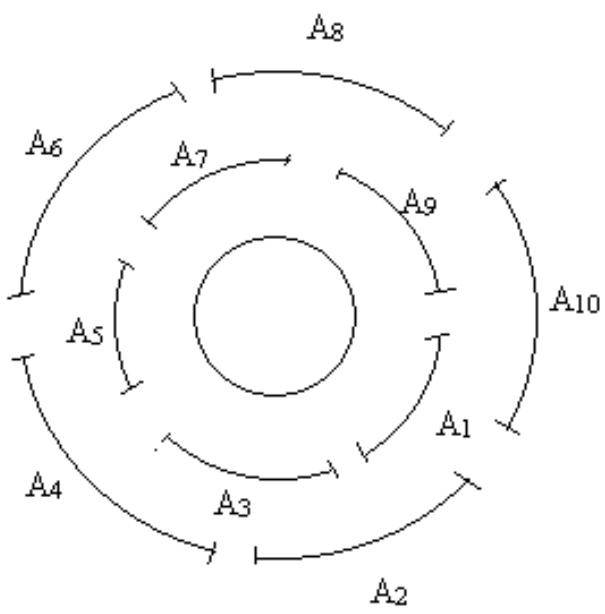

Fig. 3.3 Circular-arc family 


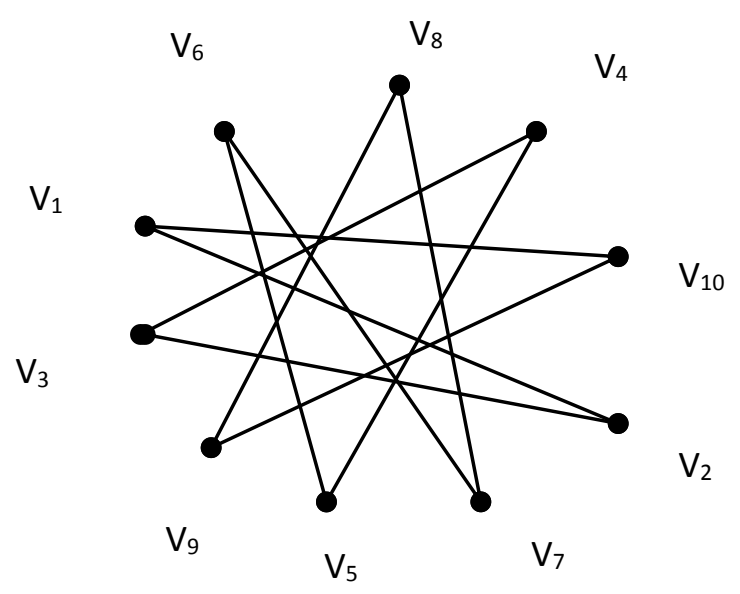

Fig.3.4 Circular-arc graph

Given circular-arc family A, there are ten arcs in which every arc intersects the next arc only.

then $v=10=3 \times 3+1$

This is of the form $v=3 a+b$, here $a=3$ and $b=1$.

Then the minimum dominating set is

$\mathrm{D}=\left\{\mathrm{v}_{3 \mathrm{i}-1}, \mathrm{v}_{\mathrm{v}} / \mathrm{i} \in \mathrm{N}, 1 \leq \mathrm{i} \leq \mathrm{a}\right\}$

$\mathrm{D}=\left\{\mathrm{v}_{3 \mathrm{i}-1}, \mathrm{v}_{\mathrm{v}} / \mathrm{i} \in \mathrm{N}, 1 \leq \mathrm{i} \leq 3\right\}$

$\mathrm{D}=\left\{\mathrm{v}_{2}, \mathrm{v}_{5}, \mathrm{v}_{8}, \mathrm{v}_{10}\right\}$ and $\gamma=4$.

$\therefore \sum_{\mathrm{v} \in \mathrm{D}} \operatorname{deg}(\mathrm{v})=\operatorname{deg}\left(\mathrm{v}_{2}\right)+\operatorname{deg}\left(\mathrm{v}_{5}\right)+\operatorname{deg}\left(\mathrm{v}_{8}\right)+\operatorname{deg}\left(\mathrm{v}_{10}\right)$

$=2+2+2+2$

$\therefore \sum_{\mathrm{v} \in \mathrm{D}} \operatorname{deg}(\mathrm{v})=8$

and (i) $\sum_{\mathrm{v} \in \mathrm{D}} \operatorname{deg}(\mathrm{v})=2 \mathrm{a}+2=2(3)+2=6+2=8$

(ii) $\sum_{\mathrm{v} \in \mathrm{D}} \operatorname{deg}(\mathrm{v})=2 \gamma=2 \times 4=8 \quad(\because \gamma=4)$

(iii) $\sum_{\mathrm{v} \in \mathrm{D}} \operatorname{deg}(\mathrm{v})=\frac{2 \mathrm{v}+4}{3}=\frac{2 \times 10+4}{3}=\frac{24}{3}=8 \quad(\because v=10)$

Hence, the theorem is proved.

\subsection{THEOREM}

Let $\mathrm{A}=\left\{\mathrm{A}_{\mathrm{i}} / \mathrm{i} \in \mathrm{N}, 1 \leq \mathrm{i} \leq \mathrm{v}\right\}, \forall \mathrm{v} \geq 3$ be any finite circulararc family inwhich every arc intersects the next arc only and $\mathrm{G}$ be a circular-arc graph corresponding to a circular-arc family A. Suppose $v=3 a+b$ where $b=0,1,2$ and $a$ is any positive integer. If $b=2$ then two cases will arise

Case (i): The minimum dominating set is

$\mathrm{D}=\left\{\mathrm{v}_{3 \mathrm{i}-1}, \mathrm{v}_{\mathrm{v}-1} / \mathrm{i} \in \mathrm{N}, 1 \leq \mathrm{i} \leq \mathrm{a}\right\}$ and $\gamma=\mathrm{a}+1$ and then the following deductions are true

(i) $\sum_{v \in D} \operatorname{deg}(v)=2 a+2($ ii $) \sum_{v \in D} \operatorname{deg}(v)=2 \gamma$

(iii) $\sum_{\mathrm{v} \in \mathrm{D}} \operatorname{deg}(\mathrm{v})=\frac{2 \mathrm{v}+2}{3}$.

Case (ii): The minimum dominating set is $\mathrm{D}=\left\{\mathrm{v}_{3 \mathrm{i}-1}, \mathrm{v}_{\mathrm{v}} / \mathrm{i} \in \mathrm{N}, 1 \leq \mathrm{i} \leq \mathrm{a}\right\}$ and $\gamma=\mathrm{a}+1$ and then the following deductions are true
(i) $\sum_{v \in D} \operatorname{deg}(v)=2 a+2\left(\right.$ ii) $\sum_{v \in D} \operatorname{deg}(v)=2 \gamma$
(iii) $\sum_{\mathrm{v} \in \mathrm{D}} \operatorname{deg}(\mathrm{v})=\frac{2 \mathrm{v}+2}{3}$.

PROOF:

Let $\mathrm{A}=\left\{\mathrm{A}_{1}, \mathrm{~A}_{2}, \mathrm{~A}_{3}, \mathrm{~A}_{4}, \ldots \ldots \ldots, \mathrm{A}_{\mathrm{v}}\right\}, \forall v \geq 3$ be any finite circular-arc family such that every arc $A_{i}$ intersects the next arc only and let $\mathrm{G}$ be a circular-arc graph corresponding to a circular-arc family $\mathrm{A}$ if there is one-to-one correspondence between the vertex set $\mathrm{V}$ and circular-arc family $\mathrm{A}$, where $\mathrm{V}=\mathrm{v}_{1}, \mathrm{v}_{2}, \mathrm{v}_{3}, \ldots \ldots ., \mathrm{v}_{v}$

Suppose $v=3 a+b$, where $b=0,1,2$ by Euclidean division lemma

If $b=2$, then $v=3 a+2 \Rightarrow v-2=3 a$

$$
\therefore \mathrm{a}=\frac{\mathrm{v}-2}{3}
$$

Then two cases will arise

Case (i): we have $\mathrm{D}=\left\{\mathrm{v}_{3 \mathrm{i}-1}, \mathrm{v}_{\mathrm{v}-1} / \mathrm{i} \in \mathrm{N}, 1 \leq \mathrm{i} \leq \mathrm{a}\right\}$ and $\gamma=\mathrm{a}$ $+1$

i.e., $D=v_{2}, v_{5}, v_{8}, \ldots \ldots \ldots . . ., v_{3 a-1}, v_{v-1}$

i.e., $\mathrm{D}=\mathrm{v}_{2}, \mathrm{v}_{5}, \mathrm{v}_{8}, \ldots \ldots \ldots . . ., \mathrm{v}_{v-3}, \mathrm{v}_{v-1}$

(i) $\sum_{\mathrm{v} \in \mathrm{D}} \operatorname{deg}(\mathrm{v})=\operatorname{deg}\left(\mathrm{v}_{2}\right)+\operatorname{deg}\left(\mathrm{v}_{5}\right)+\operatorname{deg}\left(\mathrm{v}_{8}\right)+\cdots \cdots \cdots+\operatorname{deg}\left(\mathrm{v}_{\mathrm{v}-1}\right)$

$$
\begin{aligned}
=2 & +2+2+\cdots \cdots \cdots+2+2 \\
& =2(1+1+1+\cdots \cdots \cdots+1) \\
& =2(a+1) \\
& =2 a+2
\end{aligned}
$$

$\therefore \sum_{\mathrm{v} \in \mathrm{D}} \operatorname{deg}(\mathrm{v})=2 \mathrm{a}+2$

(ii) $\sum_{\mathrm{v} \in \mathrm{D}} \operatorname{deg}(\mathrm{v})=2 \mathrm{a}+2=2(\gamma-1)+2=2 \gamma-2+2=2 \gamma \quad(\because \gamma=\mathrm{a}+1)$

$\therefore \sum_{\mathrm{v} \in \mathrm{D}} \operatorname{deg}(\mathrm{v})=2 \gamma$ 


$$
\text { (iii) } \begin{aligned}
\sum_{\mathrm{v} \in \mathrm{D}} \operatorname{deg}(\mathrm{v}) & =2 \mathrm{a}+2 \\
& =2\left(\frac{v-2}{3}\right)+2 \quad\left(\because \mathrm{a}=\frac{v-2}{3}\right) \\
& =\frac{2 v-4}{3}+2 \\
& =\frac{2 v-4+6}{3} \\
& =\frac{2 v+2}{3} \\
\therefore \sum_{\mathrm{v} \in \mathrm{D}} \operatorname{deg}(\mathrm{v}) & =\frac{2 v+2}{3}
\end{aligned}
$$

Case (ii): we have $\mathrm{D}=\left\{\mathrm{v}_{3 \mathrm{i}-1}, \mathrm{v}_{\mathrm{v}} / \mathrm{i} \in \mathrm{N}, 1 \leq \mathrm{i} \leq \mathrm{a}\right\}$ and $\gamma=\mathrm{a}+$ 1

i.e., $\mathrm{D}=\mathrm{v}_{2}, \mathrm{v}_{5}, \mathrm{v}_{8}, \ldots \ldots \ldots \ldots, \mathrm{v}_{3 \mathrm{a}-1}, \mathrm{v}_{v}$

i.e., $\mathrm{D}=\mathrm{v}_{2}, \mathrm{v}_{5}, \mathrm{v}_{8}, \ldots \ldots \ldots \ldots, \mathrm{v}_{v-3}, \mathrm{v}_{v}$

(i) $\sum_{\mathrm{v} \in \mathrm{D}} \operatorname{deg}(\mathrm{v})=\operatorname{deg}\left(\mathrm{v}_{2}\right)+\operatorname{deg}\left(\mathrm{v}_{5}\right)+\cdots+\operatorname{deg}\left(\mathrm{v}_{\mathrm{v}}\right)$

$$
\begin{aligned}
& =2+2+2+\cdots \cdots \cdots+2+2 \\
& =2(1+1+1+\cdots \cdots \cdots+1) \\
& =2(a+1) \\
& =2 a+2
\end{aligned}
$$$$
\therefore \sum_{\mathrm{v} \in \mathrm{D}} \operatorname{deg}(\mathrm{v})=2 \mathrm{a}+2
$$

(ii) $\sum_{\mathrm{v} \in \mathrm{D}} \operatorname{deg}(\mathrm{v})=2 \mathrm{a}+2=2(\gamma-1)+2=2 \gamma \quad(\because \gamma=\mathrm{a}+1)$

$\therefore \sum_{\mathrm{v} \in \mathrm{D}} \operatorname{deg}(\mathrm{v})=2 \gamma$

(iii) $\sum_{\mathrm{v} \in \mathrm{D}} \operatorname{deg}(\mathrm{v})=2 \mathrm{a}+2$

$$
\begin{aligned}
& =2\left(\frac{v-2}{3}\right)+2 \quad\left(\because \mathrm{a}=\frac{v-2}{3}\right) \\
& =\frac{2 v-4}{3}+2 \\
& =\frac{2 v-4+6}{3} \\
& =\frac{2 v+2}{3}
\end{aligned}
$$

$\therefore \sum_{\mathrm{v} \in \mathrm{D}} \operatorname{deg}(\mathrm{v})=\frac{2 v+2}{3}$

\section{PRACTICAL PROBLEM}

Let $A=\left\{A_{1}, A_{2}, A_{3}, \cdots \cdots A_{10}, A_{11}\right\}$ be the Circular-arc family and let $\mathrm{G}$ be a circular-arc graph corresponding to a circular-arc family $\mathrm{A}$ is as follows

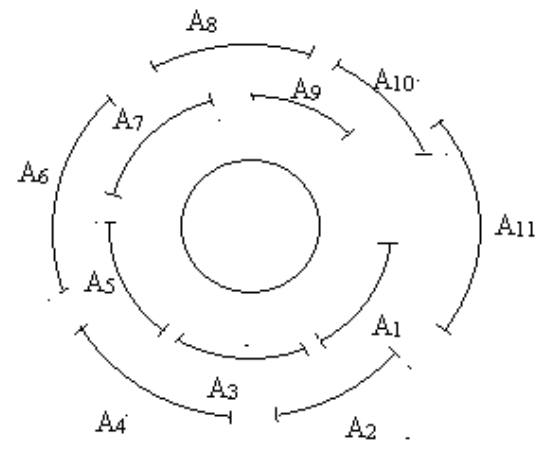

Fig. 3.5 Circular-arc family

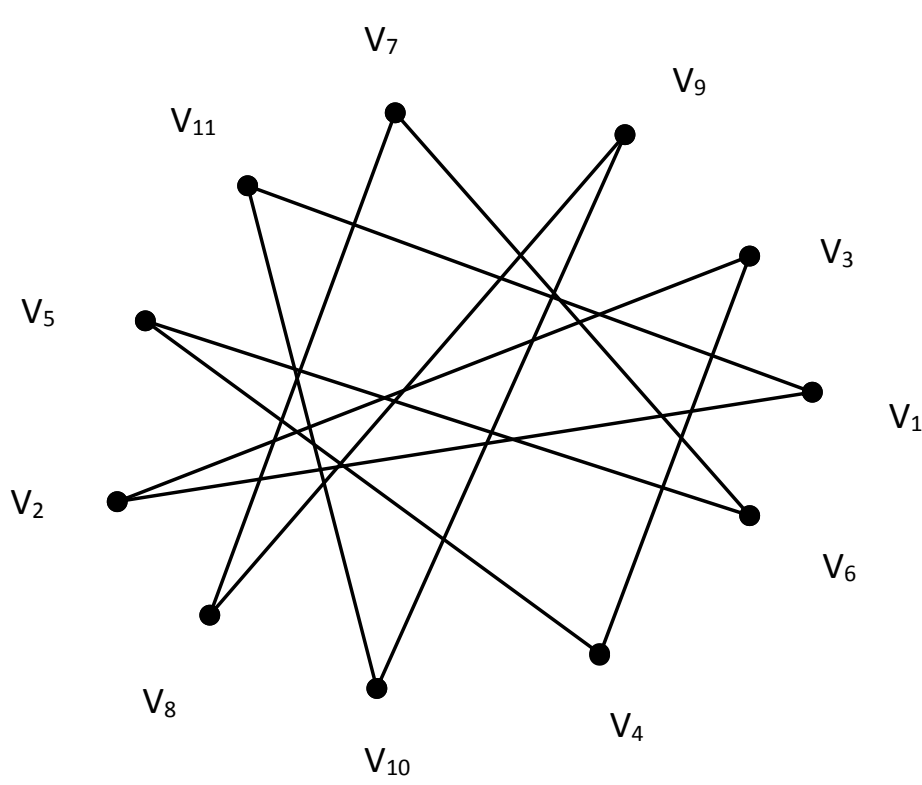

Fig.3.6 Circular-arc graph

Given circular-arc family A, there are 11 arcs in which every arc intersect the next arc only.

then $v=11=3 \times 3+2$

This is of the form $v=3 a+b$, here $a=3$ and $b=2$.

If $b=2$ then two cases will arise

Case (i): The minimum dominating set is

$$
\begin{aligned}
& \mathrm{D}=\left\{\mathrm{v}_{3 \mathrm{i}-1}, \mathrm{v}_{\mathrm{v}-1} / \mathrm{i} \in \mathrm{N}, 1 \leq \mathrm{i} \leq \mathrm{a}\right\} \\
& \mathrm{D}=\left\{\mathrm{v}_{3 \mathrm{i}-1}, \mathrm{v}_{\mathrm{v}-1} / \mathrm{i} \in \mathrm{N}, 1 \leq \mathrm{i} \leq 3\right\} \\
& \mathrm{D}=\left\{\mathrm{v}_{2}, \mathrm{v}_{5}, \mathrm{v}_{8}, \mathrm{v}_{10}\right\} \text { and } \gamma=4 .
\end{aligned}
$$


$\therefore \sum_{\mathrm{v} \in \mathrm{D}} \operatorname{deg}(\mathrm{v})=\operatorname{deg}\left(\mathrm{v}_{2}\right)+\operatorname{deg}\left(\mathrm{v}_{5}\right)+\operatorname{deg}\left(\mathrm{v}_{8}\right)+\operatorname{deg}\left(\mathrm{v}_{10}\right)$ $=2+2+2+2$

$\therefore \sum_{\mathrm{v} \in \mathrm{D}} \operatorname{deg}(\mathrm{v})=8$

and (i) $\sum_{\mathrm{v} \in \mathrm{D}} \operatorname{deg}(\mathrm{v})=2 \mathrm{a}+2=2(3)+2=6+2=8 \quad(\because \mathrm{a}=3)$

(ii) $\sum_{\mathrm{v} \in \mathrm{D}} \operatorname{deg}(\mathrm{v})=2 \gamma=2 \times 4=8 \quad(\because \gamma=4)$

(iii) $\sum_{\mathrm{v} \in \mathrm{D}} \operatorname{deg}(\mathrm{v})=\frac{2 \mathrm{v}+2}{3}=\frac{2 \times 11+2}{3}=\frac{24}{3}=8 \quad(\because v=11)$

Case (ii): The minimum dominating set is

$\mathrm{D}=\left\{\mathrm{v}_{3 \mathrm{i}-1}, \mathrm{v}_{\mathrm{v}} / \mathrm{i} \in \mathrm{N}, 1 \leq \mathrm{i} \leq \mathrm{a}\right\}$

$\mathrm{D}=\left\{\mathrm{v}_{3 \mathrm{i}-1}, \mathrm{v}_{\mathrm{v}} / \mathrm{i} \in \mathrm{N}, 1 \leq \mathrm{i} \leq 3\right\}$

$\mathrm{D}=\left\{\mathrm{v}_{2}, \mathrm{v}_{5}, \mathrm{v}_{8}, \mathrm{v}_{11}\right\}$ and $\gamma=4$.

$\therefore \sum_{\mathrm{v} \in \mathrm{D}} \operatorname{deg}(\mathrm{v})=\operatorname{deg}\left(\mathrm{v}_{2}\right)+\operatorname{deg}\left(\mathrm{v}_{5}\right)+\operatorname{deg}\left(\mathrm{v}_{8}\right)+\operatorname{deg}\left(\mathrm{v}_{11}\right)$ $=2+2+2+2$

$\therefore \sum_{\mathrm{v} \in \mathrm{D}} \operatorname{deg}(\mathrm{v})=8$

In this case also we have observed the following

(i) $\sum_{v \in D} \operatorname{deg}(v)=2 a+2\left(\right.$ ii) $\sum_{v \in D} \operatorname{deg}(v)=2 \gamma$

(iii) $\sum_{\mathrm{v} \in \mathrm{D}} \operatorname{deg}(\mathrm{v})=\frac{2 \mathrm{v}+2}{3}$.

Hence, the theorem is hold.

\section{CONCLUSION}

In this study, we focused on obtaining the some properties on sum of degrees of minimum dominating sets through the quotient ' $a$ ', domination number and the number of vertices of the circular-arc graph in different cases of the remainder $b=$ 0, 1, 2 of the Euclidean division lemma of divisor 3 for the circular-arc graph corresponding to the circular-arc family.

\section{REFERENCES}

[1] O. Ore. Theory of Graphs, Ann. Math. Soc. Colloq. Publ. 38. Providence, 1962

[2] E. J. Cockayne and S. T. Hedetniemi. Networks 7(1977), 247-261.

[3] H.B.Walikar, B.D.Acharya and E. Sampath kumar. Recent Developments in the theory of Domination in Graphs, In: MRI Lecture Notes No. 1. Allahabad 1979.

[4] S. Muthammai, M.Bhanumathi and P. Vidhya, Complimentary tree domination number of a graph.

[5] T. W. Haynes, S.T. Hedetniemi and P.J.Slater, F undamentals of domination in graphs, Marcel Dekker, Inc., NewYork (1998).

[6] T. W. Haynes, S.T. Hedetniemi and P.J.Slater, Domination in Graphs: advanced topics , Marcel Dekker, Inc., New York (1998).

[7] Kulli.V. R. and Janakiram . B, 2000,Vol.19. No.2, pp. 145156.

[8] Hedetniemi. S. T., Laskar. R. C., "Bibliography on domination in graphs and some basic definitions of domination parameters", Discrete Mathematics 86:1-3 (1990), pp. 257-277.

[9] Haynes. T. W., Hedetniemi. S. T., Slater. P. J., Fundamentals of domination in graphs, Marcel Dekker Inc., New York, 1998.

[10] Haynes. T. W., Hedetniemi. S. T., Slater. P. J., Domination in graphs: Advanced Topics, Marcel Dekker Inc., New York, 1998. 